\title{
SpS1-Instrumentation for sub-millimeter spectroscopy
}

\author{
Luis F. Rodríguez ${ }^{1}$ \\ ${ }^{1}$ Centro de Radioastronomía y Astrofísica (UNAM), Apartado Postal 3-72 (Xangari), \\ 58089 Morelia, Michoacán, México \\ email: l.rodriguez@crya.unam.mx
}

\section{Introduction}

The fields of millimeter and sub-millimeter interferometry have been developing for more than 30 years. At millimeter wavelengths the most important interferometers are the Combined Array for Research in Millimeter-Wave Astronomy (CARMA), the Plateau de Bure Interferometer (PdBI), and the Nobeyama Millimeter Array (NMA). At sub-millimeter wavelenghts, the most powerful interferometer is the SubMillimeter Array (SMA, for a detailed description, see Ho et al. 2004).

The important discoveries made by these interferometers laid the ground-work for a project without precedent in ground-based radio astronomy: the Atacama Large Millimeter Array (ALMA).

\section{The Atacama Large Millimeter Array}

ALMA is a major international collaboration between Europe, North America, East Asia, and Chile that is unique in most aspects. The interferometer will be constituted by 50 antennas of $12 \mathrm{~m}$ diameter plus the Atacama Compact Array (ACA), which will participate in the array with four $12 \mathrm{~m}$ antennas and twelve $7 \mathrm{~m}$ antennas. As a whole, ALMA will have its antennas distributed in a variable configuration that will spread from about 150 meters at its most compact, up to $16 \mathrm{~km}$. The total collecing area of the array will be larger than $6500 \mathrm{~m}^{2}$. The largest configuration will take advantage of the geography of the site (see Fig. 1), with the longest arms stretching between the hills of the site. The ACA will be located in a compact configuration, about $50 \mathrm{~m}$ in diameter, which will allow sensitive wide-field imaging and total power measurements. These configurations will provide an angular resolution from 1." 4 in its most compact state, up to 0. . 015 in its most extended state.

ALMA will cover a frequency range from 31 to $950 \mathrm{GHz}$ (10 to $0.3 \mathrm{~mm}$ ). With superconducting SIS mixers as receivers, sensitivities close to the quantum limits will be achieved. Future instruments will need to have much larger collecting areas to improve on ALMA. The correlator will be able to cover bandwidths of $16 \mathrm{GHz}$ with 4096 channels. Finally, the site is also unique; at an altitude of $5 \mathrm{~km}$ and with outstanding characteristics, the Chajnantor plains is one of the best sites in the world. A detailed review of ALMA is given by Tarenghi (2008).

\section{Sub-millimeter spectroscopy}

The sub-millimeter range is expected to be ideal for the study of thermal phenomena. Dust emission scales as $S_{\nu} \propto \nu^{2-4}$, depending on the opacity. The emission from lines will grow as $S_{\nu} \propto \nu^{5}$, and as demonstrated by the SMA, a true "forest" of molecular lines is expected. As a matter of fact, the density of detectable line emission in the sub-millimeter may turn out to be a problem, with spectral confusion being reached in some objects.

ALMA is expected to make major progress in several lines of research. Protoplanetary disks around forming stars of all masses will be resolved and their morphology and dynamics will be studied in great detail. The search for effects such as sub-Keplerian rotation and the presence of gaps and structures induced by star formation will become standard practice. Star formation is also characterized by collimated outflows of gas and ALMA will allow the investigation of these 


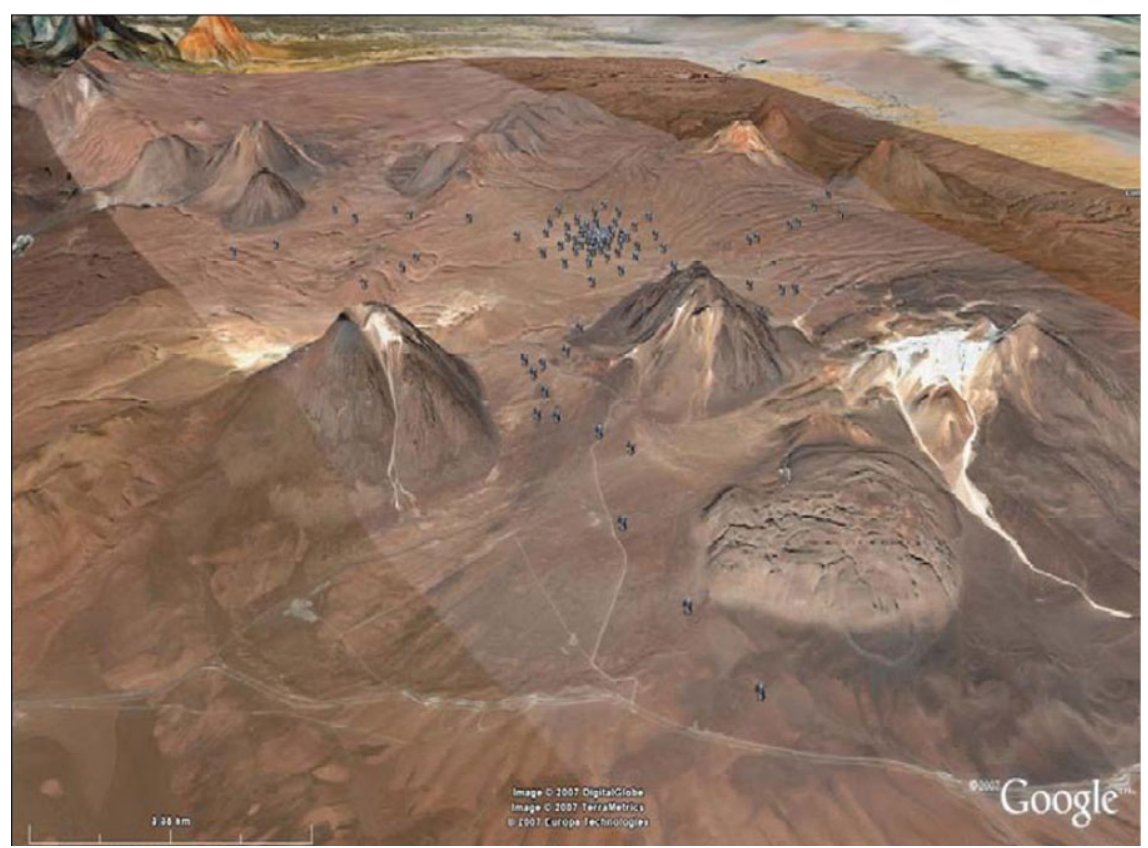

Figure 1. Google-Earth view of the ALMA site with a possible distribution of antennas superposed. This image shows the array in its most extended configuration.

phenomena with unprecedented spatial and spectral resolution. The understanding of massive star formation is now at an impasse because the sources are relatively far and small; however, ALMA will be able to produce significant advances. Evolved cool stars are surrounded by shells of molecular gas, whose chemistry is still poorly understood. Finally, the problem of galaxy formation and starburst galaxies will undergo enormous advances with ALMA. We are in the dawn of a new era of millimeter and sub-millimeter interferometry.

\section{Acknowledgements}

I thank Paul T. P. Ho and Lars Nyman for providing me with much of the material used in the presentation of this talk. I acknowledge the support of DGAPA, UNAM, and of CONACyT (México).

\section{References}

Ho, P. T. P., Moran, J. M., \& Lo, K. Y. 2004, Ap. J. 616, L1

Tarenghi, M. 2008, Astrophysics and Space Science 313, 1 